\title{
Efficacy of an ultrasonic device as a deterrent to dingoes (Canis lupus dingo): A preliminary investigation
} \author{
Griffith University, Nathan, Queensland, 4111, Australia \\ * Corresponding author. \\ Email: jason.edgar@wheb.org \\ Phone +61738757451 \\ Fax: +61738757459 \\ Total number of pages -25 \\ Total number of text pages -17 \\ Total number of tables -2 \\ Mailing address for proofs - \\ Jason Edgar \\ Wildlife-Human Ecology and Behaviour Research Group \\ c/o Associate Professor Darryl Jones \\ Australian School of Environmental Studies \\ Griffith University \\ Nathan, Queensland 4111 \\ AUSTRALIA
}

Jason P. Edgar*, Rob G. Appleby, Darryl N. Jones

Wildlife-Human Ecology and Behaviour Research Group, Australian School of Environmental Studies, 


\section{Abstract}

2 In April 2001, a nine year old child was attacked and killed by dingoes (Canis

3 lupus dingo) on Fraser Island, Queensland, Australia. The Dingo Management

4 Strategy formulated in response to this incident proposed ultrasonic deterrents

5 as one of a number of non-lethal management techniques requiring further

6 investigation. This paper describes a preliminary examination of an ultrasonic

7 deterrent to dingoes using captive animals as subjects. The commercial

8 deterrent unit tested during the study operated at ultrasonic frequencies (24-

$925 \mathrm{KHz}$ ). Two units were used simultaneously with one randomly allocated to be

10 operational during each trial. Food lures were placed in front of each unit.

11 Analysis using a Generalised Linear Mixed Model found no significant deterrent

12 effect caused by the unit. Dingoes did show a preference for eating the lure on

13 the right-hand side first, but this was not due to any deterrent effect generated

14 by the ultrasonic units. Limitations associated with the facility where the

15 experiment was conducted, coupled with a restrictive timeframe, meant that the

16 experiment did not achieve as high a level of replication as desired. It did,

17 however, provide an example of how an appropriate experimental design and

18 analytical approach can surmount many of the limitations that can beset small-

19 scale studies.

20

21 Keywords: Deterrent, Dingo, Mixed Model, Ultrasound, Wildlife-Human Interaction

23 Introduction

24 On April 30, 2001, a nine year old boy was fatally mauled by two dingoes (Canis 25 lupus dingo) at the Waddy Point camp ground on Fraser Island, Queensland, 
26 Australia. This tragic incident brought into sharp focus the inherent danger

27 present in certain interactions between humans and wildlife, particularly large 28 predators, and the need to appropriately manage situations where such 29 interactions can occur (Conover 2002). Subsequent to this attack, the 30 Queensland Parks and Wildlife Service (QPWS), the government agency 31 responsible for the management of Fraser Island, formulated the Fraser Island 32 Dingo Management Strategy (FIDMS; EPA 2001). The FIDMS recognised the importance of the Fraser Island dingo population, from both conservation and tourism standpoints, and the congruent needs of preserving dingoes whilst ensuring human safety. As well as highlighting the lack of fundamental ecological knowledge of Fraser Island dingoes and the need for further research in this regard, it also proposed the testing of a variety of non-lethal strategies for limiting and reducing the number of animals loitering around camp sites, resorts and other areas of high human use, and thereby minimising the potential for negative dingo-human interactions. Ultrasonic (i.e. above human hearing range, $>20 \mathrm{KHz}$; Stewart 1984) deterrents were identified as one of several aversive and disruptive strategies requiring further investigation (EPA 2001).

A number of studies have investigated the effectiveness of ultrasound as a deterrent in a variety of different species including rodents (e.g. Sprock et al. 1967; Greaves and Rowe 1969; Meehan 1976; Lavoie and Glahn 1977;

47 Shumake et al. 1982), deer (e.g. Curtis et al. 1995; Belant et al. 1998), bats (e.g. Hurley and Fenton 1980; van der Ree et. al. 2002), birds (e.g. Woronecki 1988), kangaroos (e.g. Bender 2001; Bender 2003), domestic cats (e.g. Mills et 
50 al. 2000, Nelson et al. 2006) and domestic dogs (e.g. Blackshaw et al. 1990).

51 As far as can be ascertained, ultrasonic deterrents have not previously been

52 tested on non-domestic canids, in captive or wild situations, although sonic (i.e.

53 within human hearing range, <20KHz; Stewart 1984) deterrents have (e.g.

54 Linhart et al. 1984 (coyotes, Canis latrans); Linhart et al. 1992 (coyotes); Breck

55 et al. 2002 (grey wolves, Canis lupus)). In almost all of the studies cited above,

56 ultrasonic deterrents demonstrated little capability of causing startle effects or

57 avoidance behaviour in target animals.

58

59 Reviews of audiogenic (i.e. sound based) deterrents by Bomford and O'Brien 60 (1990) and, to a lesser extent, Koehler et al. (1990) have criticised the lack of 61 rigour in the experimental design of many, if not most, ultrasonic experiments. 62 Inadequate replication and pseudoreplication, lack of controls, lack of 63 independence, unaccounted for temporal and spatial variation, and habituation 64 of test subjects were some of the problems identified by Bomford and O'Brien 65 (1990). The present study was designed to assess the effectiveness of a 66 commercially available ultrasonic devise on captive dingoes. While greatly 67 constrained in terms of sample size, replication and duration, one of the study's 68 major aims was to address some of these problems and demonstrate the 69 benefits of a more rigorously designed experiment in terms of overcoming 70 limitations, often using very simple and affordable methods, in combination with 71 a flexible analytical technique.

72

73 Materials and Methods 
74 The study consisted of trials with captive dingoes, as the logistics involved with

75 a similar approach involving wild individuals was prohibitive. Captive trials were

76 conducted at the facilities of the Dingo Conservation and Education Society of

77 Queensland (DCESQ), Rochedale, Queensland. Four dingoes, two males

78 (Wanji and Eenie) and two females (Dixie and Opal), each individually housed,

79 were used for this experiment. All four pens opened into a communal exercise 80 area, which served as the main experimental area.

82 As animals often respond to novel objects or situations with elevated wariness 83 (Dugatkin 2003), which in this case could have potentially confounded results 84 specifically pertaining to the dingoes responses to ultrasonic frequencies, it was 85 decided that, prior to conducting the experiment proper, dingoes would need to be familiarised with the researchers and experimental materials (e.g. non87 operation units, stands, camera equipment). Five familiarisation sessions were 88 carried out over a period of several weeks. Each session usually lasted 89 between one and two hours in duration, and consisted of researchers 90 interacting with dingoes in the communal exercise area, and, at the same time, 91 introducing dingoes to experimental materials.

93 A series of pilot assessments were also undertaken to determine the most 94 efficient experimental design. For example, as the experiment was conducted in 95 a relatively large communal exercise area which offered only one point with 96 which to power the ultrasonic units, a considerable area was outside the 97 effective range of the units. Therefore, dingoes needed to be attracted to the 
deterrent units. After pilot trials of scent-based lures failed to consistently attract dingoes, a food-based lure was chosen. This approach had the additional benefit of ascertaining whether a positive stimulus (food) could outweigh any potentially negative effects of an ultrasonic deterrent. Tinned tuna was chosen 102 as the lure due to its pungent odour. The same brand and flavour was used 103 throughout the experiment. The lure was placed on a small plastic disc connected to a short length of dowel rod which was driven into the ground so that the disc was $5 \mathrm{~cm}$ above ground level. The underside of the disc was coated with petroleum jelly to prevent ants from gaining access to the food lure.

108 The original deterrent unit that was to be used in this experiment was a Weitech 109 Transonic Electronic Yard Protector (TEYP). Measurements taken with a RION NA-20 sound pressure level (SPL) meter using a 1/3 octave filter revealed that 111 when the deterrent units were positioned in a back-to-back configuration (see 112 below), sound from the operating unit could be detected behind the unit, i.e. on 113 the non-operating unit side. As this phenomenon would invalidate the use of a 114 control unit in a two unit trial, a decision was made to replace the TEYP with a different unit. The Weitech Yard and Garden Protector (YGP) was subsequently used during the experiment. Measurements taken using the SPL meter 117 revealed no reflected sound behind the unit. When using a $20 \mathrm{KHz}$ filter, the SPL 118 meter gave an average reading of $82 \mathrm{~dB}$ directly in front of the YGP unit. Units 119 were provided for the duration of the experiment at no cost by Bird Gard 120 Australia. In an effort to gauge the acoustical properties of the unit, an ANABAT 121 II (Titley Electronics Pty. Ltd.), a device designed to detect the ultrasonic calls of 
122 microbats, was utilised (sensu Bender 2003). Through the use of a Zero-

123 Crossing Analysis Interface Module (ZCAIM), the ANABAT was connected to a 124 computer and ultrasonic signals captured and measured using the ANABAT 125 software. The YGP unit was found to produce sound in a very narrow band of 126 frequencies, between $24-25 \mathrm{KHz}$, with a cycle taking only around $100 \mathrm{~ms}$. The 127 DCESQ facility was also tested for ambient ultrasonic noise using the SPL 128 meter with a $20 \mathrm{KHz}$ filter; however no signal in the meter's operational range 129 was detected. Based upon measurements of its SPL output and confirmation of 130 stable and consistent ultrasonic frequencies, the YGP unit was considered to be 131 appropriate for testing as a potential ultrasonic deterrent.

132

133 Two ultrasonic units were mounted separately on purpose-built stands which 134 were placed back to back, enabling both units to be filmed for later analysis. 135 The use of two units simultaneously, one randomly assigned on and one off for 136 each trial was seen as the most expedient way to overcome time and facility137 related constraints. The simultaneous presentation of treatments (on) and 138 controls (off) has previously been undertaken in trials with audiogenic deterrents 139 (e.g. Shumake et al. 1982).

141 Each individual trial proceeded in the following manner: All animals were 142 removed from the enclosure and returned to their individual pens. A coin was 143 tossed to determine which unit would be on and which would be off. 144 Approximately $5 \mathrm{~g}$ (level teaspoon) of tuna was placed in front of each unit. The 145 animal indicated by a randomised draw was released by an assistant who 
146 remained seated quietly in the doorway of the pen (in an attempt to minimise

147 the influence of observer presence). Notes were made on which lure was eaten

148 first (unit on, unit off, left-hand, or right-hand sides) and whether both lures were

149 eaten as well as any novel behaviours exhibited by an individual during the trial.

150 The trial was considered complete when either both lures had been consumed

151 or after ten minutes had elapsed. The dingo was then returned to its pen. As it 152 was possible for an individual to have consecutive trials due to randomised 153 draws, a ten minute rest period was initiated between such trials in an attempt 154 to prevent a lack of interest. Even when not run consecutively, dingoes failed to 155 approach the experimental area on several occasions. As this was not 156 perceived to be due to the effect of the deterrent unit (given that they never 157 approached within its effective operating range), these trials were aborted. This 158 meant that at the end of the entire experiment a number of mistrials were 159 recorded, and the experiment was no longer equally replicated between 160 individuals. A total of four such mistrials occurred, three involving the female 161 Dixie and one involving the male Eenie, giving a total of 60 usable observations. 162 Trials were conducted over 4 consecutive days, with 16 trials attempted each 163 day (4 per dingo).

165 Statistical analysis consisted of a Generalised Linear Mixed Model (GLMM), 166 which was employed to compare both within- and between-subject variation in 167 dingo responses. GLMMs are an extension of Generalised Linear Models 168 (GLMs - see McCullagh and Nelder 1989 for a definitive discussion) that have 169 the ability to handle the high levels of correlation that are inherent in 
170 hierarchical, longitudinal, and repeated measures data through the introduction

171 of one or more random terms in the linear predictor (Breslow and Clayton 1993;

172 Lee and Nelder 1996; McCulloch 1997; Chen et al. 2003). A series of GLMM

173 models were run in the $R$ statistical software package, version 1.8.1 ( $R$

174 Development Core Team 2003), utilising the glmmPQL procedure of the MASS

175 library (Venables and Ripley 2002). This procedure attempts to fit a GLMM with

176 multivariate normal random effects in the linear predictor using Penalized

177 Quasi-Likelihood (PQL) as an approximate measure of inference (Breslow and

178 Clayton 1993; Breslow 2003).

179

180 Given the limited number of potential explanatory variables, and the restricted 181 nature of the dataset, the model chosen, using $\mathrm{ON}$ as a fixed effect, was 182 considered the most parsimonious (see below for a description of the variables 183 used in the final model). This model tests the hypothesis that the probability of 184 the right-hand food lure being consumed first is affected by which unit was 185 operating. The variables used in the model are described below.

187 The response variable used was FIRSTEAT. This was a binary variable where: $0=$ the left-hand food lure was consumed first $1=$ the right-hand food lure was consumed first

191 The explanatory variable used was ON (fixed effect). This was binary where: $0=$ the left-hand deterrent unit was operating

$$
1=\text { the right-hand deterrent unit was operating }
$$


195 The grouping term used was DINGO (random effect), which refers to the 196 repeated measurements taken on each of the four dingoes.

197

198 Results

199 The fitted (final) model is as follows:

200

201

$$
\begin{aligned}
& x=2.163465-0.8877144 \mathrm{ON} \\
& \hat{\sigma}=1.470777
\end{aligned}
$$

202 where:

203

$$
x=\ln \left(\frac{\hat{p}}{1-\hat{p}}\right)
$$

204

$205 \hat{p}$ denotes the predicted probability of 'success', and $\hat{\sigma}$ the estimated random 206 effects standard deviation.

207

208 The expression for $x$ can be solved for $\hat{p}$ giving:

209

210

$$
\hat{p}=\left(\frac{e^{x}}{1+e^{x}}\right)
$$

211

212 This gives the following results:

213

214 For $\mathrm{ON}=0, \hat{p}=0.9742753$

215 For $\mathrm{ON}=1, \hat{p}=0.939717$ 
216 The values given above are probabilities, and therefore range between 0 and 1.

217 A probability less than 0.5 indicates a 'failure', where a failure is defined as 'ate

218 the left-hand side lure first' (FIRSTEAT $=0$ ); while a probability greater than 0.5

219 indicates a 'success', where a success is defined as 'ate the right-hand side lure

220 first' (FIRSTEAT = 1). It should be borne in mind here that success and failure

221 are standard terms used when dealing with binary variables and that no other

222 connotations associated with these terms is implied. The designation of left as a

223 failure and right as a success was purely arbitrary and it could easily have been

224 reversed without altering the resultant interpretation.

225

226 The results of the model can be interpreted as follows. When $\mathrm{ON}=0$ (i.e. 'left-

227 hand unit operating'), the probability is greater than 0.5 , thus a 'success'. When

228 ON changes from 0 to 1 (i.e. 'right-hand unit operating'), the probability is again

229 greater than 0.5 , again indicating a 'success'. However it is important to note 230 that $\mathrm{ON}$ was not significant $(p=0.1570, \alpha=0.05)$, indicating that the 231 probabilities given above are statistically indistinguishable: the probability of 232 eating the right-hand lure first is the same regardless of which unit is operating.

234 While the above interpretation can be considered as the global model, because 235 a GLMM takes into account non-independence in the data there are separate 236 random intercept estimates for each dingo, thus effectively four separate 237 models. Having derived these expected values calculated by the models, they 238 can be compared to the observed values in order to evaluate the performance 239 of the model. The easiest form of comparison is to construct a set of 
240 contingency tables showing where dingoes were expected and observed to go

241 first (i.e. either left or right dependent on which unit was ON) and a simple

242 comparison made based on percentages.

243

244 For $\mathrm{ON}=0$, all four equations have a probability greater than 0.5 , indicating that 245 all dingoes would be more likely to go right first, and for $\mathrm{ON}=1$, three values 246 were higher than 0.5 , indicating that three dingoes would be more likely to go 247 right first, but one was lower indicating that one dingo would be more likely to go 248 left first. The predicted values are summarised in Table 1.

249

250 The observed values were simply calculated by tallying what side each dingo 251 went to first compared to which side the operating unit was on across the whole 252 experiment. These values are summarised in Table 2.

253

254 It can be seen that when the right unit was on $(\mathrm{ON}=1)$, the predicted and 255 observed values are close, with only a $4 \%$ skew towards eating the left-hand 256 lure first in the observed values. When the left unit was on $(\mathrm{ON}=0)$ however, 257 the model was less accurate, with a $21 \%$ skew towards eating the left-hand lure 258 first.

260 Discussion

261 The results of this study demonstrated clearly that the ultrasonic unit assessed 262 was ineffective in deterring the four dingo subjects from eating the proffered 263 food lures. Out of a total of 60 successfully completed trials, both baits were 264 consumed in 57 cases, a total of 95\%. Dixie (twice) and Eenie (once) were the 
265 only dingoes to eat a single food lure during an individual trial, and on one of 266 these occasions the uneaten lure was on the unit OFF side. This would indicate 267 that, overall, the unit did not produce enough of a deterrent effect to dissuade 268 the dingoes from eating the lures on the unit ON side. Given the criticisms 269 levelled at the analyses commonly used in the ultrasonic deterrent literature 270 (e.g. Bomford and O'Brien 1990), a GLMM was chosen to provide a more 271 robust and appropriate form of statistical analysis for these data (i.e. data that 272 are highly correlated, have unequal variance, and where there is unequal 273 replication between subjects). It should be noted that, given the small number of 274 subjects available for this experiment, the statistical power of a GLMM could be 275 brought into question. However, we are unaware of any power tests that could 276 be employed for GLMMs at this time. That aside, a GLMM is one of the few 277 tests suitable for such data. For example, Generalized Estimating Equations 278 (GEEs) are one alternative, but they are more suited to datasets with large 279 numbers of individuals and a low number of replicates (McCulloch and Searle 280 2001). One of the fundamental problems in many analyses, not only those of 281 ultrasonic deterrent experiments, is the failure to account for correlation 282 between observations, i.e. observations are not independent (Milinski 1997). 283 This lack of independence is commonly referred to as pseduoreplication 284 (Hurlbert 1984; Heffner et al. 1996; Milinski 1997). Independence is a common 285 assumption of many statistical tests which, if overlooked, can invalidate any 286 analyses done in such situations (Rao 1998). While pseudoreplication is a 287 problem under these circumstances, there are methods of dealing with such data. While redesigning the experiment is one option, the most straightforward 
method is to use a statistical test that can handle non-independence. GLMMs are one such test (Breslow and Clayton 1993; Lee and Nelder 1996; McCulloch 1997; Chen et al. 2003). One advantage of using such a test is that repeated measures enable a comparison of intra-subject variation. This allows an examination of changes over time, important in detecting behavioural processes such as habituation.

The GLMM model chosen, with ON as a fixed effect, while not yielding 297 statistically significant results, did appear to provide a relatively good fit to the 298 data, as seen by the comparison of observed versus expected values. Overall, 299 the model predicted that regardless of which unit was ON (left or right), the 300 dingoes would eat the right-hand food lure first more than $90 \%$ of the time, 301 slightly higher than the observed value of $78 \%$. The ability to break down the 302 model and identify the effect of each subject shows that it was primarily Opal 303 and Wanji, and to a lesser extent Eenie, that drove this trend. Dixie was the only 304 subject in the model to always display a preference for eating the unit OFF side 305 lure first. The probability of her eating the right-hand lure first when the left-hand 306 unit was ON was $63 \%$, while the probability of her eating the left-hand lure first 307 when the right-hand unit was ON was $58 \%$ (recall that the probabilities given 308 are always for eating the right-hand lure first (FIRSTEAT $=1)$, therefore the 309 probability of eating the left-hand lure first is $1.00-0.42)$. In both cases this was 310 only marginally more than what would be expected by random chance (i.e. $31150 \%$ ) and is therefore not enough to suggest any noticeable deterrent effect 312 generated by the unit, especially given that ON was not significant. 
314 While it may appear that the preference three of the four subjects (Wanji, Eenie and Opal) showed for eating the right-hand side lure first might be attributable to some effect of the deterrent unit, the cause of this trend was most likely the

317 location of the release points of the individual animals from their respective 318 pens. Opal's release point meant that she had to pass the right-hand unit on her 319 way to the left-hand unit, so not surprisingly she always went to the right-hand side first. Eenie and Wanji displayed some interesting behaviour in that, when 321 released from their pens, they often both chose to walk along the concrete path 322 running along the edge of their pens, rather than walking straight forwards 323 across an open grassed area towards the units. This path-following behaviour 324 often meant that both animals arrived at the right-hand unit first, as the path 325 lead almost directly towards that side. Eenie, and to a lesser extent Wanji, 326 showed a lessening of this path-following in the latter half of the experiment.

328 Why both Eenie and Wanji displayed this behaviour is uncertain. It may have 329 been some apprehension due to the presence of strange objects and people in 330 their enclosure that caused them to stay close to their familiar pens which 331 gradually declined as the experiment went on, although this seems unlikely. 332 Both animals generally showed little fear of the units or the stands they were 333 mounted on during familiarisation sessions; rather, they were initially inquisitive 334 about these novel objects. It may have been due to the social contention 335 between these two males. A series of agonistic displays between these males were witnessed throughout the two month period over which the experiment 
337 was conducted. On numerous occasions, both males scent marked nearby 338 bushes after or during a trial. It may be that both were detecting scent trails left 339 by one another on the concrete paths and followed these trails to investigate 340 what the other was up to. Dingoes in the wild have been shown to travel on

341 paths and roads in preference to crossing open ground or moving through thick 342 vegetation (Mahon et al., 1998), which may also provide some explanation for 343 the similar behaviour demonstrated by Wanji and Eenie. Importantly, this bias 344 for going to the right-hand side first was in no way deemed to be due to any 345 effect generated by the deterrent unit.

347 While this bias for eating the right-hand food lure first was potentially 348 problematic in terms of exploring the efficacy of the deterrent, given the 349 conditions under which the experiment was conducted, there was little could be 350 done to alleviate it. The layout of the enclosure with its various natural features 351 such as gardens, rocks, logs, trees, etc., and restrictive access to a power 352 source meant that the location chosen to site the units was unavoidable. That 353 being said, the right-hand bias in no way invalidates the experiment. On the contrary, the fact that the unit in no way deterred the dingoes from eating the right-hand lure first as often as they did (78\%) is evidence of its lack of 356 effectiveness as a dingo deterrent. The right-hand lure operated in $51.7 \%$ of 357 trials $(n=31)$, slightly more frequently than the left-hand unit $(48.3 \%, n=29)$.

358 Thus once again there was no evidence that the unit was in any way influencing 359 the dingoes' behaviour. Finally, confidence in the performance of the experiment, the analysis chosen, and the results obtained is bolstered by the 
361 simple fact that dingoes ate both food lures (including the lure on the unit ON side) on all but several occasions.

364 In addition to providing an initial investigation of the effectiveness of a specific 365 dingo deterrent, this project also attempted to provide an example of how a 366 more rigorous study could be undertaken to assess the efficacy of such a 367 device, in the presence of many potential limitations. The approach taken here 368 demonstrated that, even with limited resources, such an experiment can still be 369 conducted relatively rigorously. While the findings may be constrained due to 370 the small number of dingo subjects available and the necessity to conduct the

371 experiment with captive dingoes, the experiment itself provides useful 372 guidelines as to how appropriate experimental designs and associated analyses 373 facilitate greater confidence in determining the efficacy of such a deterrent.

375 While not discounting the obvious caution required in interpreting these findings, 376 this study concurs with others that found no measurable deterrent effect (e.g. 377 Lavoie and Glahn 1977; Woronecki 1988; Belant et al. 1998; Mills et al. 2000; 378 Bender 2001; van der Ree et al. 2002; Bender 2003), suggesting that 379 ultrasound may be of little use as a deterrent for dingoes, and focus should now 380 be shifted towards investigations of other potential, non-lethal management 381 techniques. 


\section{Acknowledgements}

Mike Noble of the DCESQ graciously provided full access to his captive dingoes and facilities to allow this study to take place. The ultrasonic deterrent units used during this project were supplied by Jim Savage of Bird Gard Australia. James McBroom of Griffith University advised on statistical analysis and interpretation.

\section{References}

Belant JL, Seamans TW, Tyson LA (1998) Evaluation of electronic frightening devices as white-tailed deer deterrents. Proc Vertebr Pest Conf 18:107-110

Bender H (2001) Deterrence of kangaroos from roadways using ultrasonic frequencies: Efficacy of the Shu Roo. Department of Zoology, University of Melbourne, Melbourne

Bender H (2003) Deterrence of kangaroos from agricultural areas using ultrasonic frequencies: Efficacy of a commercial device. Wildl Soc Bull 31:10371046.

Blackshaw JK, Cook GE, Harding P, Day C, Bates W, Rose J, Brahmam D (1990) Aversive response of dogs to ultrasonic, sonic and flashing light units. Appl Anim Behav Sci 25:1-8 
Bomford M, O’Brien PH (1990) Sonic deterrents in animal damage control: A review of device tests and effectiveness. Wild Soc Bull 18:411-422

Breck SW, Williamson R, Niemeyer C, Shivik JA (2002) Non-lethal radio activated guard for deterring wolf depredation in Idaho: Summary and call for research. Proc Vertebr Pest Conf 20:223-226

Breslow NE (2003) Whither PQL? UW Biostatistics Working Paper Series, Working Paper 192

Breslow NE, Clayton DG (1993) Approximate inference in generalized linear mixed models. J Am Stat Assoc 88:9-25

Chen MH, Ibrahim JG, Shao QM, Weiss RE (2003) Prior elicitation for model selection and estimation in generalized linear mixed models. J Stat Plan Infer $111: 57-76$

Conver M (2002) Resolving human-wildlife conflicts: The science of wildlife damage management. Lewis Publishers, Boca Raton

Curtis PD, Fitzgerald C, Richmond ME (1995) evaluation of the Yard Gard ultrasonic yard protector for repelling white-tailed deer. Proc East Wildl Dam Cont Conf 7:172-176 
Dugatkin LA (2003) Principles of animal behaviour. WW Norton and Co. Inc, London

Environmental Protection Agency (EPA) (2001) Fraser Island dingo management strategy. Environmental Protection Agency, Queensland Government, Brisbane

Greaves JH, Rowe FP (1969) Responses of confined rodent populations to an ultrasound generator. J Wildl Manage 33:409-417

Heffner RA, Butler MJ, Reilly CK (1996) Pseudoreplication revisited. Ecology $77: 2558-2562$

Hurlbert SH (1984) Pseudoreplication and the design of ecological field experiments. Ecol Monogr 54:187-211

Hurley S, Fenton MB (1980) Ineffectiveness of fenthion, zinc phosphide, DDT and two ultrasonic rodent repellers for control of populations of little brown bats (Myotis lucifugus). Bull Environ Contam Toxicol 25:503-507

Koehler AE, Marsh RE, Salmon TP (1990) Frightening methods and devices/stimuli to prevent mammal damage - A review. Proc Vertebr Pest Conf $14: 168-173$ 
Lavoie GK, Glahn JF (1977) Ultrasound as a deterrent to Rattus norvegicus. J Stored Prod Res 13:23-28

Lee Y, Nelder JA (1996) Hierarchical generalized linear models. J R Stat Soc B 58:619-678

Linhart SB, Dasch GJ, Johnson C, Roberts JD, Packham CJ (1992) Electronic frightening devices for reducing coyote predation on domestic sheep: Efficacy under range conditions and operational use. Proc Vertebr Pest Conf 15:386-392

Linhart SB, Sterner RT, Dasch GJ, Theade JW (1984) Efficacy of light and sound stimuli for reducing coyote predation upon pastured sheep. Prot Ecol 6:75-84

Mahon PS, Banks PB, Dickman CR (1998) Population indices for wild carnivores: A critical study in sand-dune habitat, south western Queensland. Wild Res 25:11-22

McCullagh P, Nelder JA (1989) Generalized linear models. 2nd Edition. Chapman and Hall, London

McCulloch CE (1997) Maximum likelihood algorithms for generalized linear mixed models. J Am Stat Assoc 92:162-170 
McCulloch CE, Searle SR (2001) Generalized, linear, and mixed models. John Wiley and Sons Inc., New York

Meehan AP (1976) Attempts to influence the feeding behaviour of brown rats using ultrasonic noise generators. Int Pest Control 18:12-15

Mills DS, Bailey SL, Thurstans RE (2000) Evaluation of the welfare implications and efficacy of an ultrasonic 'deterrent' for cats. Vet Rec 147:678-680

Milinski M (1997) How to avoid seven deadly sins in the study of behavior. Adv Study Behav 26:159-179

Nelson SH, Evans AD, Bradbury, RB (2006) The efficacy of an ultrasonic cat deterrent. Appl Anim Behav Sci 96:83-91

R Development Core Team (2003) R: A language and environment for statistical computing. R Foundation for Statistical Computing, Vienna, Austria Online: <http://www.R-project.org>

Rao PV (1998) Statistical research methods in the life sciences. Brooks/Cole Publishing Company, Pacific Grove, California

Shumake SA, Kolz AL, Crane KA, Johnson RE (1982) Variables affecting ultrasound repellency in Philippine rats. J Wildl Manage 46:148-155 
Sprock CM, Howard WE, Jacob FC (1967) Sound as a deterrent to rats and mice. J Wild Manage 31:729-741

Stewart JL (1984) Acoustics in pest control. Av-Alarm Corp., Oregon

van der Ree R, Bender $\mathrm{H}$, Nelson J (2002) The effectiveness of ultrasound at deterring grey-headed flying-foxes from roosting in the Fern Gully, Royal Botanic Gardens, Melbourne - A pilot study. Research Report Number 7. Australian Research Centre for Urban Ecology, Royal Botanic Gardens Melbourne, Melbourne

Venables WN, Ripley BD (2002) Modern applied statistics with S. 4th Edition. Springer, New York

Woronecki PP (1988) Effect of ultrasonic, visual, and sonic devices on pigeon numbers in a vacant building. Proc Vertebr Pest Conf 13:266-272 


\section{Tables and Figures}

Table 1 Expected values derived from model

(Percentages in italics are relative percentages calculated across rows)

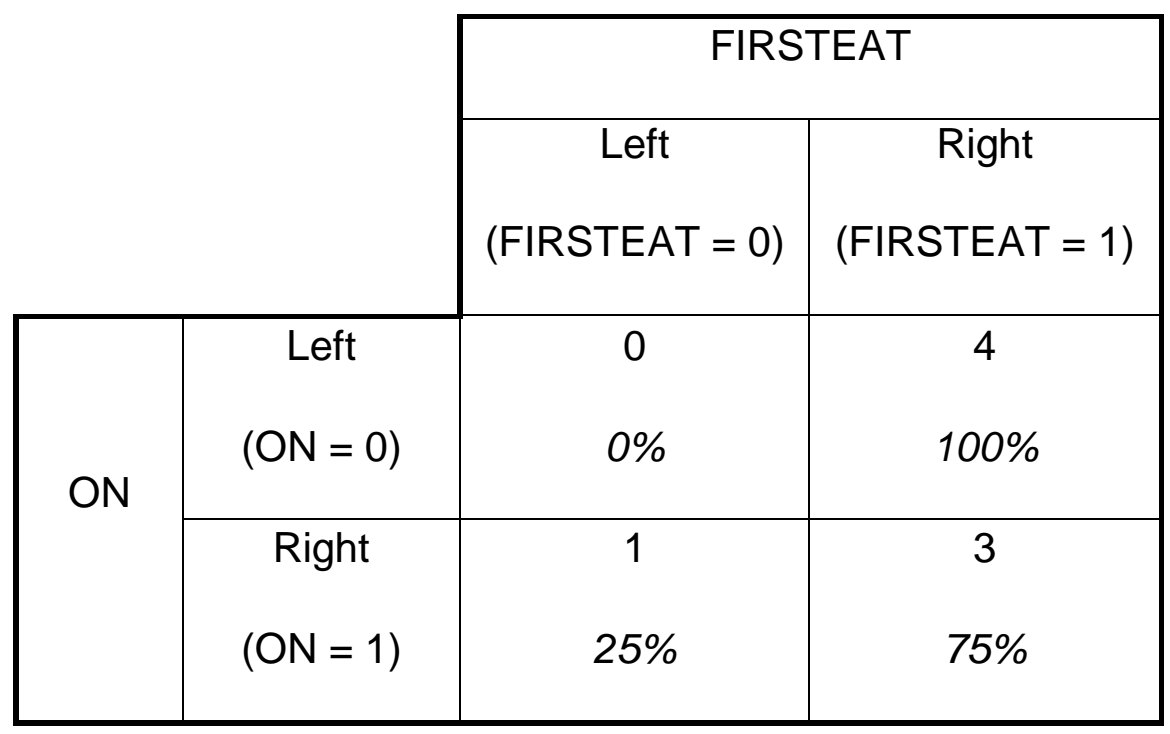


Table 2 Observed values from dataset

(Percentages in italics are relative percentages calculated across rows)

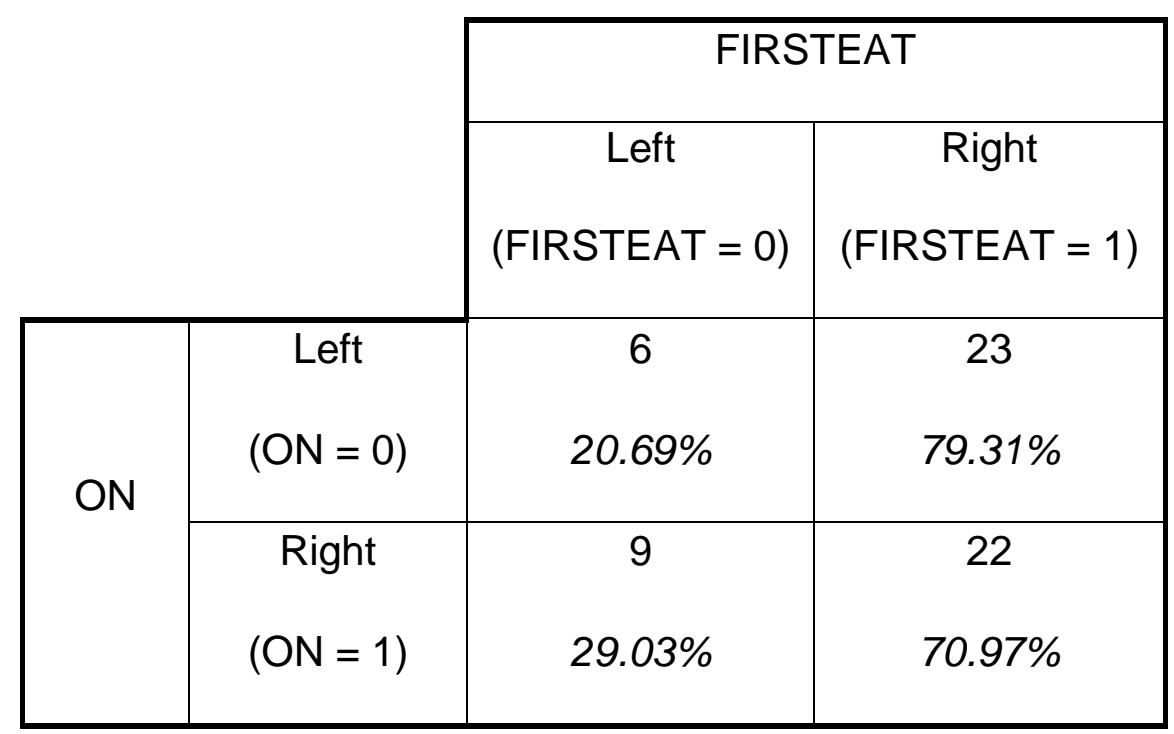

